\title{
Quantum Stress Focusing in Descriptive Chemistry
}

\author{
Jianmin Tao, ${ }^{1,2}$ Giovanni Vignale, ${ }^{2}$ and I. V. Tokatly ${ }^{3,4}$ \\ ${ }^{1}$ Theoretical Division and CNLS, Los Alamos National Laboratory, Los Alamos, New Mexico 87545, USA \\ ${ }^{2}$ Department of Physics, University of Missouri-Columbia, Columbia, Missouri 65211, USA \\ ${ }^{3}$ European Theoretical Spectroscopy Facility (ETSF), Departamento de Fisica de Materiales, Universidad del Pais Vasco UPV/EHU, \\ 20018 San Sebastian, Spain \\ ${ }^{4}$ Moscow Institute of Electronic Technology, Zelenograd, 124498 Russia
}

(Received 24 December 2007; published 22 May 2008)

\begin{abstract}
We show that several important concepts of descriptive chemistry, such as atomic shells, bonding electron pairs, and lone electron pairs, may be described in terms of quantum stress focusing, i.e., the spontaneous formation of high-pressure regions in an electron gas. This description subsumes previous mathematical constructions, such as the Laplacian of the density and the electron localization function, and provides a new tool for visualizing chemical structure. We also show that the full stress tensor, defined as the derivative of the energy with respect to a local deformation, can be easily calculated from density functional theory.
\end{abstract}

DOI: 10.1103/PhysRevLett.100.206405

PACS numbers: 71.15.Mb, 31.15.E-, 71.45.Gm

Atomic shell structure, electron pair domains, $\pi$-electron subsystems, etc., are common concepts in descriptive chemistry and play a significant role in modern electronic structure theory. These concepts help us to visualize the bonding between atoms in terms of small groups of localized electrons (e.g., two electrons of opposite spin in a simple covalent bond) and therefore play an important role in predicting new molecular structures and in describing structural changes due to chemical reactions.

A precise quantitative description of small groups of localized electrons has been sought for a long time [1,2], but none of the solutions proposed thus far is completely satisfactory. The natural candidate - the electronic density $n(\mathbf{r})$ - has a clear physical meaning, but fails to reveal quantum mechanical features such as atomic shell structure [3] or the localization of electron pairs of opposite spin in a covalent bond. The Laplacian $\nabla^{2} n$ of the density [1,4] provides a better way to visualize molecular geometry, but lacks a clear physical significance and fails [5] to reveal the atomic shell structure of heavy atoms. Recently, a very useful indicator of electron localization has been constructed [5] from the curvature of the (spherically averaged) conditional pair probability function $P_{\sigma \sigma}\left(\mathbf{r}, \mathbf{r}^{\prime}\right)$ (the probability of finding an electron of spin $\sigma$ at $\mathbf{r}$ given that there is another electron of the same spin orientation at $\mathbf{r}^{\prime}$ ) evaluated at $\mathbf{r}=\mathbf{r}^{\prime}$. In the simplest approximation this curvature is proportional to

$$
D_{\sigma}(\mathbf{r})=\tau_{\sigma}-\frac{\left|\nabla n_{\sigma}\right|^{2}}{8 n_{\sigma}}
$$

where $\tau_{\sigma}(\mathbf{r})=\sum_{l} \frac{1}{2}\left|\nabla \psi_{l \sigma}(\mathbf{r})\right|^{2}$ is the noninteracting kinetic energy density of $\sigma$-spin electrons (atomic units $m=$ $\hbar=e^{2}=1$ are used throughout $), \psi_{l \sigma}(\mathbf{r})$ are the occupied Kohn-Sham orbitals of spin $\sigma$, and $n_{\sigma}$ is the $\sigma$-spin electron density $\left(n=n_{\uparrow}+n_{\downarrow}\right)$. From $D_{\sigma}$ one constructs the electron localization function (ELF) [5]

$$
\eta_{\sigma}(\mathbf{r})=\frac{1}{1+\left(D_{\sigma} / \tau_{\sigma}^{\mathrm{TF}}\right)^{2}},
$$

where $\tau_{\sigma}^{\mathrm{TF}}=(3 / 10)\left(6 \pi^{2}\right)^{2 / 3} n_{\sigma}^{5 / 3}$ is the Thomas-Fermi kinetic energy density of $\sigma$-spin electrons. The ELF provides excellent visualization of atomic shells as well as a quantitative description of valence shell electron pair repulsion theory [6] of bonding and it has been recently extended to excited and time-dependent states [7]. Nevertheless, it has two defects: (i) it remains a mathematical construction of dubious physical significance [8] and (ii) it is difficult to calculate beyond [9] the lowest-order approximation [Eq. (1)], and for this reason it can hardly be applied to strongly interacting systems.

In this Letter we propose a more physical way of looking at atomic shells and bonds based on the idea of quantum stress focusing, by which we mean the spontaneous formation of regions of high and low pressure in the electron gas. We also show that, unlike ELF, the quantum stress tensor can be easily calculated beyond the lowest-order approximation within the framework of density functional theory [10,11]. A proper mapping of the stress tensor [12] may be the long-sought solution to the problem of visualizing molecular structure.

The existence of high and low pressure regions in an atom is nontrivial in view of the fact that the electrostatic potential created by the nuclei has no maxima or minima. To understand how pressure maxima and minima can arise we must recall a few basic concepts from the mechanics of continuum media. First, we introduce the stress tensor $[13,14] p_{i j}(\mathbf{r})$ - a symmetric rank-2 tensor with the property that $p_{i j}$ is the $i$ th component of the force per unit area acting on an infinitesimal surface perpendicular to the $j$ th axis [15]. The divergence of this tensor, $\sum_{j} \partial_{j} p_{i j}(\mathbf{r})\left(\partial_{j}\right.$ being the derivative with respect to $r_{j}$, the $j$ th component of $\mathbf{r}$ ), is the $i$ th component of the force per unit volume 
exerted on an infinitesimal element of the electron gas by the surrounding electrons. For convenience, in the following we will exclude from the stress tensor the electrostatic Hartree contribution, which is treated as an external field on equal footing with the field of the nuclei.

Next, we separate $p_{i j}$ into a hydrostatic pressure part, which is isotropic, and a shear part, which is traceless:

$$
p_{i j}(\mathbf{r})=\delta_{i j} p(\mathbf{r})+\pi_{i j}(\mathbf{r}), \quad \operatorname{Tr} \pi_{i j}=0 .
$$

Here $p(\mathbf{r}) \equiv \frac{1}{3} \operatorname{Tr} p_{i j}(\mathbf{r})$ is the quantum pressure, i.e., the derivative of the internal energy with respect to a local deformation that changes the volume of a small element of the electron liquid without changing its shape. Similarly, the shear stress $\pi_{i j}(\mathbf{r})$ is the derivative of the internal energy with respect to a local deformation that changes the shape of a small element of the liquid, without changing its volume. In a quantum system, these local deformations are implemented through a local change in the metrics [see Eq. (11) and Ref. [14] for details]. In the ground state of an electronic system the forces arising from the divergence of $p_{i j}$ must exactly balance the external force exerted by the nuclei. In other words, the stresses satisfy the equilibrium condition

$$
-n(\mathbf{r}) \partial_{i} v(\mathbf{r})=\partial_{i} p(\mathbf{r})+\sum_{j} \partial_{j} \pi_{i j}(\mathbf{r}),
$$

where $v(\mathbf{r})$ is the external potential due to the nuclei plus the Hartree potential.

Equation (4) is not very useful if we do not have explicit expressions for $p$ and $\pi_{i j}$. However, certain approximations can be made. For example, replacing the exact quantum pressure by the quasiclassical Fermi pressure $p^{\mathrm{TF}}(\mathbf{r})=\frac{2}{5}\left(3 \pi^{2}\right)^{2 / 3} n^{5 / 3}$ and neglecting the shear term yields the well-known Thomas-Fermi equation for the equilibrium density. Because the electrostatic potential $v(\mathbf{r})$ in an atom does not admit maxima and minima, we immediately see that this approximation (as any approximation that neglects $\pi$ ) cannot yield local maxima or minima in the pressure. In the special case of the spherical Thomas-Fermi atom this implies that the density and the pressure are both monotonically decreasing functions of the radial distance $r$ : the shell structure is absent.

The situation changes radically when the shear stress is included. Now it is possible for the hydrostatic force $\partial_{i} p$ to vanish because the electrostatic force $n \partial_{i} v$ can be balanced by the shear force $\sum_{j} \partial_{j} \pi_{i j}$. It is therefore possible to have maxima or minima in the pressure. We may picture the regions of high pressure as regions which are hard to compress, because of the high energy cost of bringing the particles closer together against the "exchangecorrelation (XC) hole." These "maximally compressed regions" quite naturally correspond to the atomic shells of descriptive chemistry.

We now show that regions of maximum and minimum pressure do occur in spherical atoms, with peaks corre- sponding to the $K, L, M, \ldots$ shells of the standard shell model. To see this we do not need to go any further than the lowest-order approximation for the stress tensor, which has the form

$$
p_{i j}^{\mathrm{S}}=\frac{1}{2} \sum_{l \sigma}\left(\partial_{i} \psi_{l \sigma}^{*} \partial_{j} \psi_{l \sigma}+\partial_{j} \psi_{l \sigma}^{*} \partial_{i} \psi_{l \sigma}\right)-\frac{1}{4} \delta_{i j} \nabla^{2} n,
$$

where the sum runs over the occupied Kohn-Sham orbitals. For an atom of spherical symmetry this further simplifies to

$$
p_{i j}^{\mathrm{S}}=p^{\mathrm{S}} \delta_{i j}+\pi^{\mathrm{S}}\left(\frac{r_{i} r_{j}}{r^{2}}-\frac{\delta_{i j}}{3}\right),
$$

where $p^{\mathrm{S}}$ is the noninteracting pressure given by

$$
p^{\mathrm{S}}=\frac{1}{3} \sum_{l \sigma}\left|\nabla \psi_{l \sigma}\right|^{2}-\frac{1}{4} \nabla^{2} n
$$

and

$$
\pi^{\mathrm{S}}=\sum_{l \sigma}\left(\left|\partial_{r} \psi_{l \sigma}\right|^{2}-r^{-2}\left|\partial_{\theta} \psi_{l \sigma}\right|^{2}\right)
$$

To graphically represent the noninteracting pressure $p^{\mathrm{S}}$, we define the dimensionless quantity $\tilde{p}^{\mathrm{S}}=p^{\mathrm{S}} / p^{\mathrm{TF}}$. Since this ratio diverges both at a nucleus (with a positive sign) and in the density tail (with a negative sign) [16], we find it convenient to plot the function

$$
L(\mathbf{r})=\frac{1}{2}\left(1+\frac{\tilde{p}^{\mathrm{S}}}{\sqrt{1+\left(\tilde{p}^{\mathrm{S}}\right)^{2}}}\right)
$$

which has values in the range $(0,1)$, with $L=1$ corresponding to $\tilde{p}^{\mathrm{S}}=\infty$ and $L=0$ corresponding to $\tilde{p}^{\mathrm{S}}=$ $-\infty$. Figures 1 (a) -1 (d) show conclusively the existence of
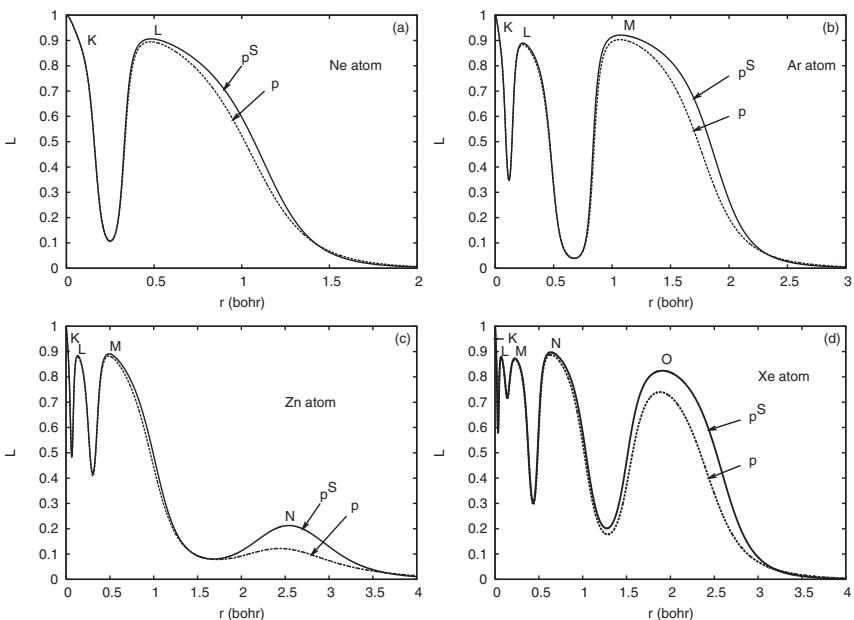

FIG. 1. Indicators of electron localization as functions of the radial distance $r$ constructed, respectively, from the noninteracting and interacting pressures $p^{\mathrm{S}}$ and $p$ [Eq. (9)] for the (a) $\mathrm{Ne}$, (b) Ar, (c) Zn, and (d) Xe atoms. The XC part of the pressure is evaluated with the GGA functional of Ref. [18]. The maxima of the pressure reveal electronic shells, indicated as usual by the capital letters $K, L, M, \ldots$. 
peaks and troughs of the noninteracting pressure (solid curves), each of which defines a spherical surface on which the gradient of $p^{\mathrm{S}}$ vanishes. Since $p^{\mathrm{TF}}$ is a monotonically decreasing function, the oscillatory behavior of these graphs is entirely due to $p^{\mathrm{s}}$ : in particular, the maxima and minima of $L$ are very close to the maxima and minima of $p^{\mathrm{S}}$. As discussed above, we interpret the peaks in pressure as quantum shells. Figure 2 shows the behavior of $p^{\mathrm{S}}$ and $\pi^{\mathrm{S}}$ as functions of $r$, and the inset depicts schematically the distribution of shear forces in the atom. Observe that the minima of the shear stress are in correspondence with the maxima of the pressure, and vice versa. This is natural, because the gradients of $p$ and $\pi$, (i.e., approximately, the bulk force and the shear force) are large, and must therefore have opposite signs in order to produce a net force that balances the relatively weak nuclear attraction. The fact that the shell is located at the minimum of the shear stress implies that the shear force has opposite signs on the two sides of the shell, thus "locking" the shell into position.

Two more points should be made about Eq. (7) for the pressure. The first is that the Laplacian of the density appears in it prominently, as a universal component of the pressure: this explains a posteriori the partial success of the Laplacian as an indicator of shell structure. More interestingly, the ELF [Eq. (2)] is also closely related to the pressure. Indeed, the noninteracting pressure includes a "bosonic" contribution, $p_{0}$, which is obtained from Eq. (7) by putting all the electrons in the lowest energy orbital $\psi_{0 \sigma}$ :

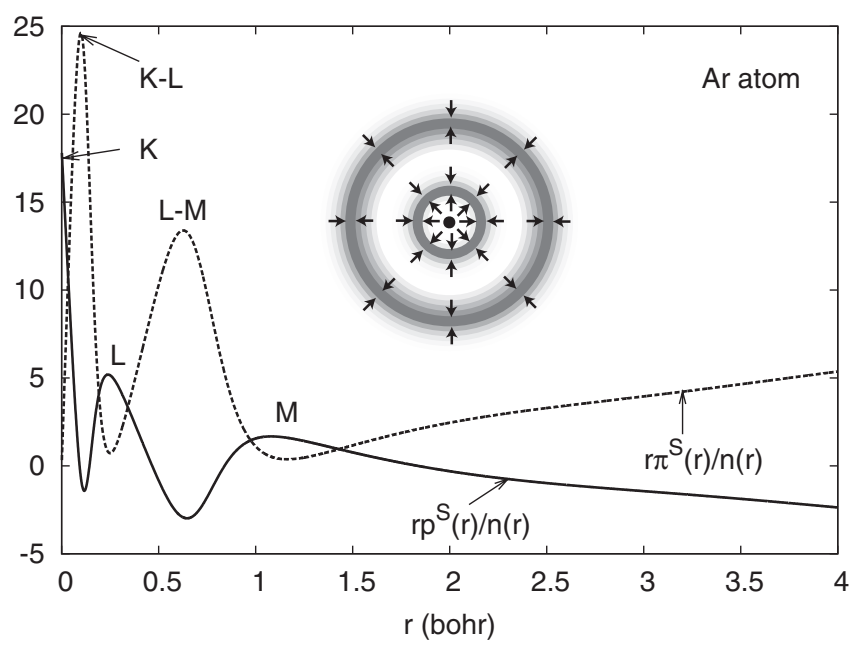

FIG. 2. Noninteracting pressure $\left(p^{\mathrm{S}}\right)$ and shear stress $\left(\pi^{\mathrm{S}}\right)$ in Ar. The peaks in $p^{\mathrm{S}}$ identify the position of the shells and the peaks in $\pi^{\mathrm{S}}$ define the boundary between the shells. Inset: Distribution of stresses in the atom. The gray scale indicates the pressure distribution with dark (light) regions corresponding to high (low) value of the pressure indicator $L$ [Eq. (9)]. The arrows indicate the direction of the shear force $(2 / 3) \nabla \pi+(2 / r) \pi$. Observe how the shells are "squeezed" by shear forces pointing in opposite directions.

$$
p_{0 \sigma}=(1 / 3)\left[\left|\nabla n_{\sigma}\right|^{2} /\left(4 n_{\sigma}\right)-(3 / 4) \nabla^{2} n_{\sigma}\right] .
$$

The excess pressure $p_{\mathrm{ex} \sigma} \equiv p_{\sigma}^{\mathrm{S}}-p_{0 \sigma}(\mathbf{r})$ is called "Pauli pressure" (since it is generated by the Pauli exclusion principle, which forces the occupation of higher energy orbitals) and is easily seen to coincide with $D_{\sigma}$, the curvature of the conditional probability function [Eq. (1)] and main ingredient of the ELF. Physically, high electronic pressure means that it is difficult to bring the particles closer together, which implies that the XC hole surrounding the electron is "deep": this leads to a high value of the ELF.

One of the attractive features of our proposal is the relative ease with which strong electron-electron interactions can be included in the calculation of the stress tensor. Already $p^{\mathrm{S}}$ includes some interaction effects through the Kohn-Sham orbitals [10]. To include additional XC effects we observe that in density functional theory (DFT) the XC energy is a functional not only of the density $n$, but also, tacitly, of the metrics $g_{i j}$ of the space [14]: $E_{\mathrm{XC}}=$ $E_{\mathrm{XC}}\left[n, g_{i j}\right]$. In conventional applications of DFT, one works with fixed Euclidean metrics $g_{i j}=\delta_{i j}$, so there is no need to emphasize the dependence on the metrics. Here, however, we are interested in small deformations, which can be described by the coordinate transformation [17] $\boldsymbol{\xi}(\mathbf{r})=\mathbf{r}-\mathbf{u}(\mathbf{r})$, where $\mathbf{u}$ is an infinitesimal nonlinear function of $\mathbf{r}$. The transformation from $\mathbf{r}$ to $\boldsymbol{\xi}$ changes the density from $n(\mathbf{r})$ to, up to first order in $\mathbf{u}, \tilde{n}(\boldsymbol{\xi})=$ $n(\boldsymbol{\xi})+u_{i} \partial_{i} n(\boldsymbol{\xi})$ (notice that $i$ here are Cartesian indices with the convention that repeated indices are summed over), and the metric tensor from the Euclidean value $\delta_{i j}$ to

$$
g_{i j} \equiv\left(\partial r_{\alpha} / \partial \xi_{i}\right)\left(\partial r_{\alpha} / \partial \xi_{j}\right)=\delta_{i j}+\partial_{i} u_{j}+\partial_{j} u_{i} .
$$

Since the XC energy cannot depend upon the arbitrary choice of coordinates we must have $E_{\mathrm{XC}}\left[n, \delta_{i j}\right]=$ $E_{\mathrm{XC}}\left[\tilde{n}, g_{i j}\right]$, which for an arbitrary infinitesimal transformation implies

$$
n \partial_{i} \frac{\delta E_{\mathrm{XC}}}{\delta n}=\partial_{j}\left\{\delta_{i j} n \frac{\delta E_{\mathrm{XC}}}{\delta n}-2 \frac{\delta E_{\mathrm{XC}}}{\delta g_{i j}}\right\},
$$

where the functional derivative with respect to $n$ is taken at constant $g_{i j}$ and vice versa. Since the left-hand side of this equation is the $\mathrm{XC}$ force density, the right-hand side must be the divergence of the XC stress tensor. This leads to the result

$$
p_{i j}^{\mathrm{XC}}=\delta_{i j} n v_{\mathrm{XC}}-2 \frac{\delta E_{\mathrm{XC}}}{\delta g_{i j}},
$$

where $v_{\mathrm{XC}} \equiv \delta E_{\mathrm{XC}} / \delta n$ is the XC potential, and the functional derivative is evaluated at $g_{i j}=\delta_{i j}$. This important result shows that we may calculate the $\mathrm{XC}$ contribution to the stress tensor from a knowledge of the XC energy as a functional of density and metrics. It turns out that semilocal XC functionals $[18,19]$ are of a form that can be easily generalized to include a nontrivial metrics. 
For example, consider the generalized gradient approximation (GGA) for a spin-unpolarized system. The standard form of this functional is $E_{\mathrm{XC}}[n]=\int d \mathbf{r} e_{\mathrm{XC}}(n, s)$, where $s=|\nabla n| /\left(2 k_{F} n\right)$ is the reduced density gradient. Going to curvilinear coordinates we immediately obtain the generalized form

$$
E_{\mathrm{XC}}\left[\tilde{n}, g_{i j}\right]=\int d \boldsymbol{\xi} \sqrt{g(\bar{\xi})} e_{\mathrm{XC}}(\tilde{n}, \tilde{s}),
$$

where $g \equiv \operatorname{det}\left(g_{i j}\right)$ is the determinant of the metric tensor, $\tilde{n}(\boldsymbol{\xi}) \equiv n(\mathbf{r}(\boldsymbol{\xi}))$ is the density in the new coordinates, and $\tilde{s}=\sqrt{g^{i j} \partial_{i} \tilde{n} \partial_{j} \tilde{n}} /\left(2 \tilde{k}_{F} \tilde{n}\right)$, where $\tilde{k}_{F}=\left(3 \pi^{2} \tilde{n}\right)^{1 / 3}$ and $g^{i j}=$ $\left(g^{-1}\right)_{i j}$. Using the identity $\delta g=g g^{i j} \delta g_{i j}=-g g_{i j} \delta g^{i j}$, we evaluate the functional derivative of $E_{\mathrm{XC}}\left[\tilde{n}, g_{i j}\right]$ with respect to $g_{i j}$, and then substitute it into Eq. (13). We finally obtain

$$
p_{i j}^{\mathrm{XC}}=\delta_{i j}\left(n v_{\mathrm{XC}}-e_{\mathrm{XC}}\right)-\frac{\partial_{i} n \partial_{j} n}{2 k_{F} n|\nabla n|} \frac{\partial e_{\mathrm{XC}}}{\partial s} .
$$

The trace of this yields the $\mathrm{XC}$ pressure

$$
p^{\mathrm{XC}}=n v_{\mathrm{XC}}-e_{\mathrm{XC}}-\frac{s}{3} \frac{\partial e_{\mathrm{XC}}}{\partial s},
$$

which exactly recovers the uniform-gas limit [13].

In Figs. 1(a)-1(d) we have also plotted the normalized pressure $L(r)$ including the XC correction (dashed curves), which is calculated by the GGA of Ref. [18]. In the core region of the atom, where the density is high, $p^{\mathrm{XC}}$ is negligible. In the valence region or outmost shell, where the density is low, the XC contribution becomes relatively more important, compared to the kinetic part, so the difference is noticeable. In the density tail, both $p^{\mathrm{S}}$ and $p^{\mathrm{XC}}$ decay exponentially, causing the difference to be small. It is possible, however, and indeed quite likely, that XC contributions become much more important in strongly correlated systems. This remains a subject for future investigations [20].

In conclusion, we have found that the analysis of the quantum stress field reveals important features of the electronic structure of atoms. Although we have considered only atoms thus far, there is every reason to expect that stress focusing will also occur in molecules in correspondence of electron pair domains, such as the ones that are commonly associated with single and multiple covalent bonds and with the so-called lone-pair regions of the molecule. More information is likely to emerge from the complete study of the stress tensor (its eigenvalues and principal axes) and its topology. With visualization techniques [12] for tensor fields employed in such diverse fields as engineering, geology, medicine, and biomechanics, we may visualize the process of bond breaking and formation in chemical reactions.
We acknowledge valuable discussions with C. A. Ullrich and P. L. de Boeij. This work was supported by DOE under Grant No. DE-FG02-05ER46203, and by DOE under Contract No. DE-AC52-06NA25396 and Grant No. LDRD-PRD X9KU at LANL (J.T).

[1] R. F. W. Bader, Atoms in Molecules-A Quantum Theory (Oxford University, Oxford, 1990).

[2] R.J. Gillespie and I. Hargittai, The VSEPR Model of Molecular Geometry (Allyn \& Bacon, Boston, 1991).

[3] P. W. Ayers and R. G. Parr, Int. J. Quantum Chem. 95, 877 (2003).

[4] R. F. W. Bader and H. Essen, J. Chem. Phys. 80, 1943 (1984).

[5] A.D. Becke and K.E. Edgecombe, J. Chem. Phys. 92, 5397 (1990).

[6] R.J. Gillespie, Molecular Geometry (Van Nostrand Reinhold, London, 1972).

[7] T. Burnus, M. A. L. Marques, and E. K. U. Gross, Phys. Rev. A 71, 010501(R) (2005).

[8] A. Savin, THEOCHEM 727, 127 (2005).

[9] E. Matito, B. Silvi, M. Duran, and M. Solá, J. Chem. Phys. 125, 024301 (2006).

[10] W. Kohn and L. J. Sham, Phys. Rev. 140, A1133 (1965).

[11] R. G. Parr and W. Yang, Density Functional Theory of Atoms and Molecules (Oxford University, Oxford, 1989).

[12] T. Delmarcelle and L. Hesselink, IEEE Comput. Graph. Appl. 13, 25 (1993).

[13] O. H. Nielsen and R. M. Martin, Phys. Rev. B 32, 3780 (1985).

[14] I. V. Tokatly, Phys. Rev. B 71, 165104 (2005); 71, 165105 (2005); 75, 125105 (2007).

[15] $p_{i j}(\mathbf{r})$ is formally defined as the functional derivative of the internal energy with respect to a local strain $u_{i j}(\mathbf{r})$, arising from a deformation in which each particle is displaced from $\mathbf{r}_{n}$ to $\mathbf{r}_{n}+\mathbf{u}\left(\mathbf{r}_{n}\right)$, with $u_{i j} \equiv\left(\partial_{i} u_{j}+\right.$ $\left.\partial_{j} u_{i}\right) / 2$. This deformation corresponds to a local change in metrics [see Eq. (11)] which modifies the Hamiltonian according to the formulas given in Ref. [14].

[16] The positive divergence at a nucleus is caused by the Laplacian of the density, which diverges as $1 / r$ for $r \rightarrow$ 0 . The negative divergence in the density tail $(r \rightarrow \infty)$ reflects the negative value of $p^{\mathrm{S}}$ in the nonclassical regions where the wave function decays exponentially: $p^{\mathrm{S}} \stackrel{r \rightarrow \infty}{\rightarrow}$ $-4 n\left|\epsilon_{\max }^{\mathrm{KS}}\right| / 3$, where $\epsilon_{\max }^{\mathrm{KS}}$ is the highest occupied KohnSham orbital energy of the atom.

[17] J. Tao, G. Vignale, and I. V. Tokatly, Phys. Rev. B 76, 195126 (2007).

[18] J. P. Perdew, K. Burke, and M. Ernzerhof, Phys. Rev. Lett. 77, 3865 (1996).

[19] J. Tao, J. P. Perdew, V. N. Staroverov, and G. E. Scuseria, Phys. Rev. Lett. 91, 146401 (2003).

[20] With the present interpretation [see discussion below Eq. (10)], $p^{\mathrm{XC}}$ of Eq. (16) can also be used to refine the calculation of the conventional ELF. 